\title{
An Improved and Cost Effective Radio Frequency based Object Detection System
}

\author{
Okegbile Samuel Dayo \\ Department of Computer \\ Science and Engineering, \\ Obafemi Awolowo University, \\ lle-Ife, Nigeria
}

\author{
Segun Aina \\ Department of Computer \\ Science and Engineering, \\ Obafemi Awolowo University, \\ lle-Ife, Nigeria
}

\author{
Ogunleye Gbenga K. \\ Department of Computer \\ Science and Engineering, \\ Obafemi Awolowo University, \\ lle-lfe, Nigeria
}

\begin{abstract}
The need to develop a system capable of locating misplaced objects in homes has resulted in many efforts toward developing object detection system. While few systems are already available in markets, these systems have been largely unaffordable and less efficient due to high market price, short range of coverage among others. This paper attempts to design and implement an object detection system that when produced in large quantity can be affordable by the majority and provide solutions to the short-range problem experienced in the existing systems, thereby eliminating the stress of finding lost items in homes. Existing object detection systems were carefully studied to understand the design process, the cost implication and why the systems have been less efficient. Radio frequency based detection model capable of detecting lost items was designed and implemented using various components such as encoder, decoder, transmitter, receiver and buzzer. The performance of the designed system was evaluated using detection based metric. The developed object detection system was further compared with the existing systems available in markets using the cost in market and the technology used. The result showed that the proposed system is cost effective and will be affordable to people living in low income countries.
\end{abstract}

\section{General Terms}

Wireless Communications, Data communication and computer networks.

\section{Keywords}

Tagged item; Electromagnetic spectrum; Encoder; Decoder; RF Transmitter; RF receiver.

\section{INTRODUCTION}

The continuous evolution of Information Technology (IT) has led to dramatic increase in its usage in various homes such as in lighting control [1], outdoor lawn irrigation [2], controlling various kitchen appliances [3], object tracking and detection [4] and securing of systems to provide more convenience and comfort in various homes. Object tracking and detection is however one of the advancement of technology in every societies that still needs serious attention. An object detecting system comprises a physical object recognition unit which recognizes physical objects in a given environment such as homes and offices [5]. Various technologies have been adopted in tracking or detecting misplaced items. These technologies includes infrared, passive Radio Frequency Identification Device (RFID) which covers a short range, active RFID and Bluetooth which are both expensive to implement when a wider range is considered. While existing literature have considered radio frequency as another possible technology which can be adopted, this has not been widely explored in detecting lost items.
Radio Frequency (RF) systems make use of signals in a form of electromagnetic wave, which makes up a portion of the Electromagnetic (EM) spectrum. The EM spectrum encompasses all forms of waves ranging from audible frequencies such as the ubiquitous $60 \mathrm{~Hz}$, through the standard radio bands which include Amplitude Modulation (AM), Frequency Modulation (FM), Television (TV) channels and other RF bands. The spectrum continues through infrared, visible, and ultra-violet light, to higher forms of EM energy like X-rays, Gama-rays, and cosmic rays [6]. This work therefore focuses on designing a cost-effective object detecting system using RF technology in order to reduce the stress of finding lost objects in homes.

The misplacement of various objects or items such as wallet, keys and every other portable home device has been one of the major challenges faced by the owners of such items. While existing literature have considered the use of infrared, RFID [7] and Bluetooth [8] in locating or detecting lost items in homes, these methods have been largely less efficient as a result of high cost of implementation, short range of coverage and line of sight issue. Although, implementation of ODS has received a reasonable level of attention in the literature, to the best of the authors knowledge, there exists little or no consideration on developing a cost effective object detection system that can be easily affordable to people living in low income countries. In countries where feeding poses a lot of issues, it will be practically unreasonable to spend tens of dollars to procure an ODS. This made the use of the existing ODS limited to only developed countries as most people living in developing countries may not be able to afford such. This work will therefore design and implement a cost effective object detecting system using RF technology thereby making the technology available to majority if not all.

The holy grail of this project is to produce an object detecting system that when produced in large quantity can be affordable by the majority and provide solutions to the short-range problem experienced in the existing systems, thereby eliminating the stress of finding lost items in homes. The design will of course improve on the existing traditional methods of detecting misplaced objects which is inadequate and time consuming.

The remainder of this paper is as follow; Section two presented the discussion of the related works. Section three presented the system architecture and the model developed while also discussing the methods used in the implementation of the proposed ODS. Section four however presented the detailed of the components used in the implementation and the performance evaluation. Section five concluded the work and presented few recommendations for future research. 


\section{RELATED WORKS}

The authors in [9] considered an object tracking system with the aim of developing a system capable of identifying the location of a tracked person-portable object such as a security tracking bag and money pack. The authors adopted the use of Global Positioning Satellite (GPS) System and radio frequency identification tag. An event detector sends a signal by way of the communicator to the central processor with the signal indicating the occurrence of a predetermined event. This work aimed at discovering methods of detecting and tracking lost objects in a given location. The developed system makes use of GPS which makes it practically impossible to be widely adopted in most developing countries. This effort is similar to the work reported in [4]. In [4], the authors considered Global Positioning System (GPS) and Global System for Mobile Communication (GSM) bluetooth enabled tracking system. The user is made to choose the object misplaced from the mobile application. The pseudorandom code is generated and radio signals are broadcasted. The matching signal from the receiver end is found. When the item is found, the buzzer in the system gets activated and starts ringing. The user gets alert and finds the item. If the item is not located, the user is asked if there is need to switch on the GPS system. If the GPS option is selected, the system maps to the required address in order to tacked the misplaced object. Internet connectivity is however needed for the system to be used in locating objects at a far distance.

An indoor tracking system based on Bluetooth technology was developed in [10]. The work developed a system capable of tracking indoor objects. The developed system was made up of a central monitoring system, the device being tracked and Bluetooth based access points. The access points were implemented applications on Personal Computers (PCs) with bluetooth radio adapters connected to them. Access points only resend the message sent by the device being tracked. The access points are placed in the room such that their positions are known in the $\mathrm{XY}$ plane. The central monitoring system knows the initial location of all the devices being tracked. When the device moves considerably, the monitoring system sounds alarm displaying the new location, in coordinate form of the device. Each device being tracked is connected to the three access points. A bluetooth enabled tracker is attached to the device being tracked. The tracker communicates with the Central Monitoring System (CMS) and the three selected access points. The connection strength was used to determine the distance when tracking objects. Three access points were however used during the project implementation which makes the idea cost ineffective to implement.

The use of bluetooth technology has also been considered in other literature. For example, the authors in [4] considered an indoor Bluetooth-based Positioning System which aimed at determining the actual location of the items connected to it. The triangulation method combined with least square estimation was used to predict the position of the terminal. The functional dependence between the received Radio Signal Strength Indicator (RSSI) and the distance was obtained by a well fitted polynomial approximation. A test was carried out and the system was limited by a distance less than $8 \mathrm{~m}$ between the access point and the mobile device. This work was carried out using a stationed bluetooth access points to determine the position of an object. The work presented in [11] also implemented a bluetooth based secured key finder system using android Operating System (OS). The authors in [11] however focused on developing a system useful in locking and unlocking vehicle door as well as switching the vehicle on/off.

A support system for finding lost objects using spotlight was developed in [7]. The developed system was useful in finding lost objects indoor. RFID and ultrasonic sensor were used in order to enhance the accuracy of the system in finding lost items indoor. The system illuminates the position by using a moving light obstructed by others. The measured values from the two devices were fused using a particle filter. The particle filter was used not only to fuse two types of measured values but also to enable the addition of new position detection devices to the system easily. The moving light was used for notifying the detected position to the user. This device is normally used in stage lighting. The pan and tilt of the moving light were calculated backward by detecting the angle of each robot arm from the position of its hand. Results showed that, the use of light has an advantage compared with sound. Active RFID was used to realize the design in order to cover a wider range.

In [12], the authors developed a system capable of monitoring the presence of items while issuing an alert if an item is not detected. The work investigated various methods and systems that can be used in monitoring the presence of an item, which starts by determining the item for a given user; determining the proximity between the monitoring device and the lost item, based on the determined context, wherein the proximity is given by one or more proximity requirements, each requirement indicating a required proximity between the monitoring device and the lost item and a notification process corresponding to each proximity requirement. Monitoring proximity of the item based on the received signal from the system is facilitated with the aid of a notification which can be in any defined form which helps to determine the next line of action to be taken.

The authors in [13] also developed an object detection and tracking robot using android, arduino and open computer vision $(\mathrm{CV})$. The work aimed to implement a robot which can detect motion and follow an object at the same time. This can be considered as a prototype for developing advanced models based on automated home security systems. The project was implemented with the help of arduino board integrated with bluetooth support with Direct Current (DC) motors for the steering and movement of the robot. The application interface for the steering was implemented using open CV libraries and an application for android. The system process seems costly with the use of robot.

\section{METHODOLOGY}

The overview of the system architecture is presented in Fig. 1. To ease the implementation process, the system was divided into the two sub-systems;

a) Base station

b) Tagged items

a) Base station sub-system - This part of the system coordinates the activity of the system. It is made up of RF transmitter module and an encoder.

b) Tagged item sub-system - This sub-system contains any object that can be easily misplaced in homes. Example of those items include keys, TV remote control, wallet etc. This subsystem is made up of the RF receiver module, Decoder and Buzzer. 


\subsection{The System Model}

In order to satisfy the engineering requirement, the system model was designed using proteus simulation and electronic modelling tool. The model consists of a RF system which has a connection with the tag. The model was designed in such a way that it can be adapted in various homes without any modifications. Many objects can be tagged. The work however tags only one object due to limited funding. The tag can be removed and placed on any object since it does not have any direct connection with the objects. The block diagram and the model design are shown is shown in Fig. 2 and Fig. 3 respectively.
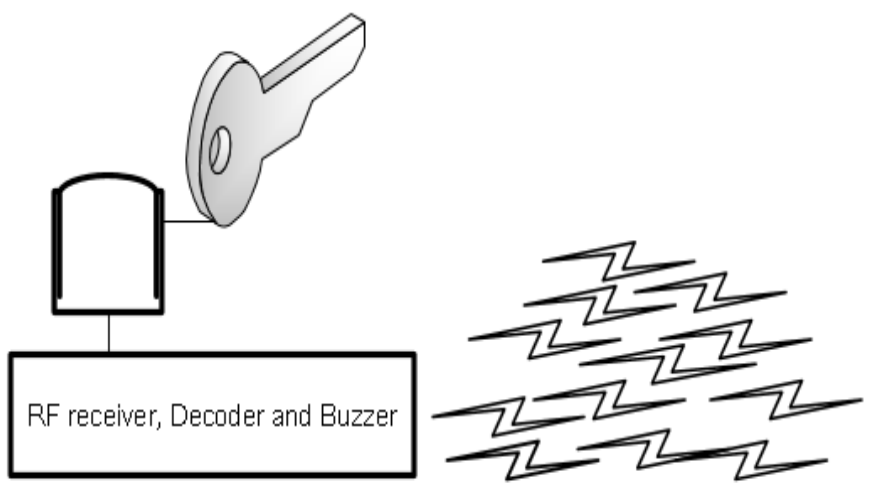

Radio Waves

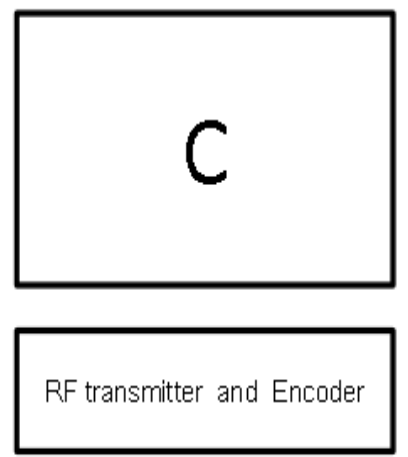

Fig. 1: Overview of System Architecture

\subsection{Working Principle}

The working principle describes how various components interrelate and communicate to produce a complete system. The HT12E encoder IC converts the 4-bit data from the 4 data pins that are connected to the power into serial data. This serial data is sent to RF transmitter. The transmitter begins the RF communication and accept the data in electrical form, modifying the same using a modulation technique to encode the data into the radio signal. The transmitter is also responsible for determining the power level of the signal. Next, an antenna collects the signal received from the transmitter and directs the RF waves away from the antenna. As the RF waves move away from the transmitting antenna, it moves towards another antenna attached to the receiver. The receiver takes the received signal from the antenna which is in form of serial data, this serial data is sent to HT12D decoder
IC which converts it into 4-bit parallel data and translates the modulated signals. Once the connection is established between the receiver and the transmitter, the buzzer connected to the receiver produces a sound which will help in locating the misplaced object.

\section{IMPLEMENTATION AND TESTING}

The designed system aimed at achieving the following criteria;

i. The system must be able to effectively create a connection between the base substation and the tag.

ii. The tag should produce a sound with the aid of the buzzer when the connection is established.

iii. The sound produced should be enough to locate the misplaced tagged object.

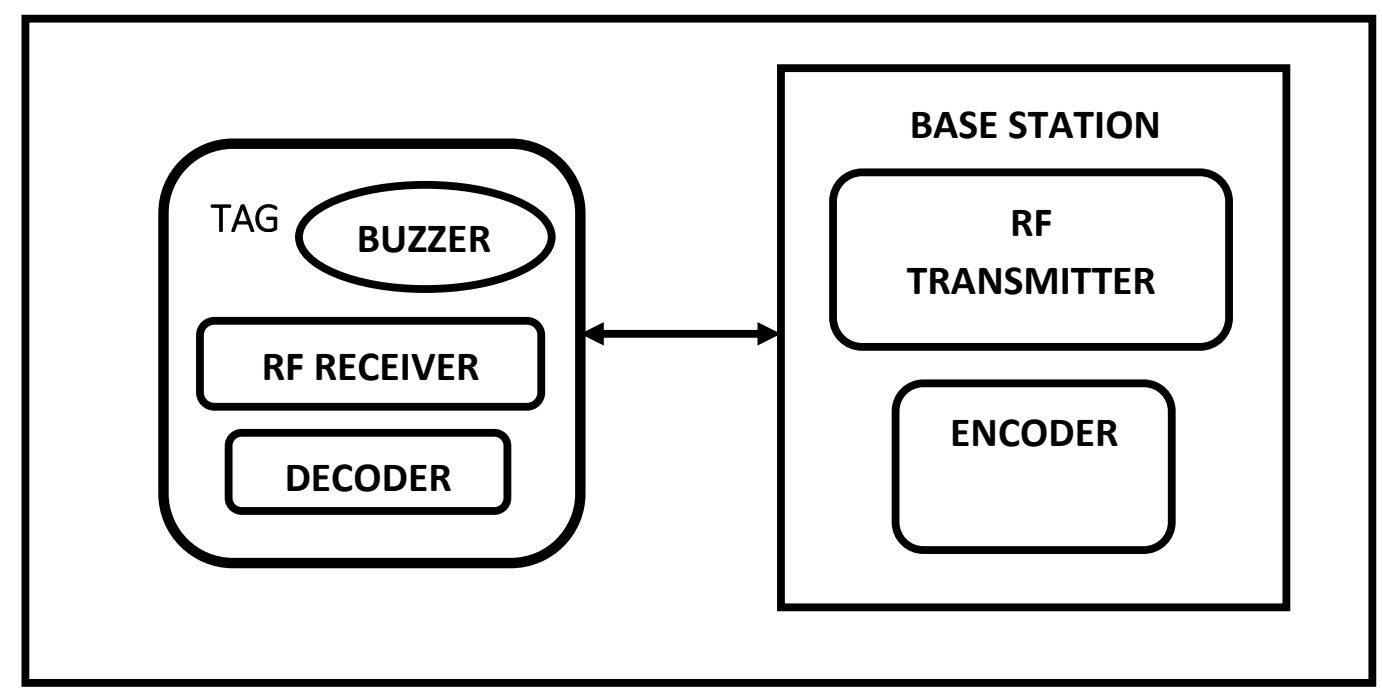

Fig. 2: Block diagram of the system model 


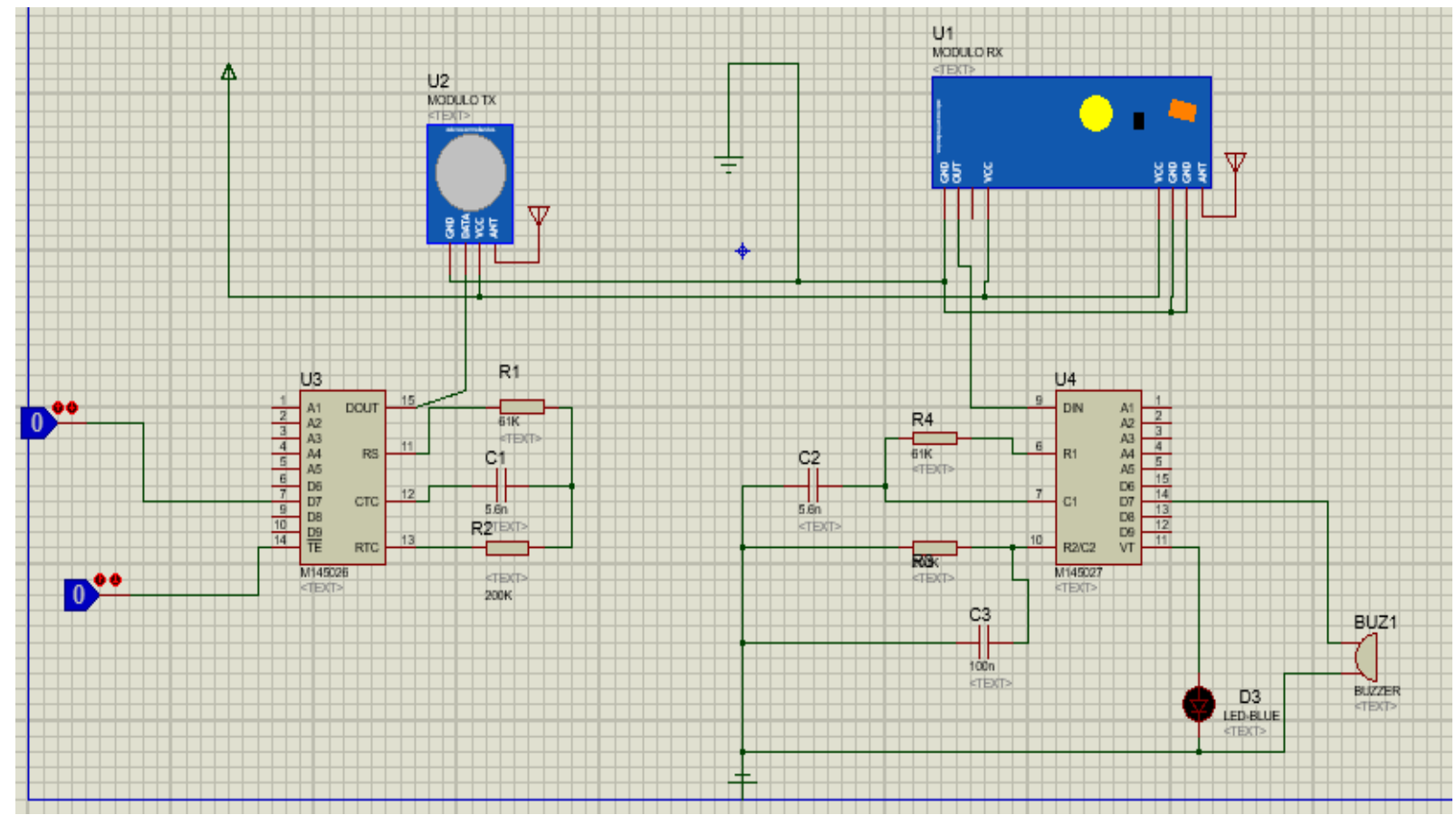

Fig. 3: Model design of the proposed ODS

\subsection{Implementation Process}

In order to achieve the criteria stated above, the system was properly implemented and carefully packaged. The proper components descriptions and details of the implementation are hereby presented;

\subsubsection{The radio frequency transmitter}

The RF transmitter Vcc was powered with a 9V Direct Current (DC) battery and the output pin was interfaced with the HT12E encoder. The RF transmitter generates analog signals as its output and therefore required an Analog-toDigital converter (ADC). The HT12E encoder ADC was initialized and then used to convert the signal from analog signal to digital signal. The RF transmission normally occurs at the rate of $1 \mathrm{Kbps}-10 \mathrm{Kbps}$.

\subsubsection{HT12E Encoder}

HT12E requires working with a low signal received from the transmitter on the transmission enable pin. After receiving the low signal, the HT12E starts the transmission of 4 data bits and the output cycle will repeats based on the status of the transmission enable pin in the IC. If the transmission enable pin retains the low signal, the cycle repeats as long as the low signal in the transmission enable pin exists. The encoder IC will be in standby mode if the transmission enable pin is disabled and thus the status of this pin was necessary for encoding process. The address of these bits was set appropriately through pin A0 - A7 and the same scheme was used in decoders to retrieve the signal bits.

\subsubsection{HT12D Decoder}

HT12D decoder will normally be in standby mode initially i.e., oscillator is disabled and a HIGH on DIN pin activates the oscillator. Thus the oscillator will be active when the decoder receives data transmitted by the encoder. The decoder device starts decoding the input address and data. The decoder matches the received address three times continuously with the local address given to pin A0 - A7. If all matches, data bits are decoded and output pins D8 - D11 are activated. This valid data is indicated by making the pin Valid Transmission (VT) HIGH. Pins D8-D11 helps to relay the decoded data to the RF receiver. This will continue till the address code becomes incorrect or no signal is received.

\subsubsection{The radio frequency receiver}

The RF receiver has 4 pins. Only 3 of these pins were used during implementation, these are Ground (GND), Vcc (5V) and one Data pin. The RF receiver data pin was connected to pin DIN of HT12D decoder for the transfer of data between the two components. The receiver was powered with a $3.3 \mathrm{~V}$ battery.

\subsubsection{Crystal oscillator}

The crystal oscillator used in this work has two pins. These are OSC1 and OSC2 pins connected in parallel to the HT12 encoder and the other end connected in parallel to ground. This was necessary in order to ensure frequency stability of the generated pulse.

\subsubsection{The buzzer}

The work used a buzzer which has 2 pins. These two pins are Vcc and Ground. The Vcc pin of the buzzer was connected to the pin D7 of HT12D decoder. The GND pin was also connected to the GND which the decoder was connected to. The buzzer produces a sound when it receives data from the decoder i.e. when a connection is established between the RF receiver and the RF transmitter.

\subsubsection{Power supply}

A $9 \mathrm{~V}$ battery and $3.3 \mathrm{~V}$ was used to power the system. The $9 \mathrm{~V}$ battery was used to power the base station subsystem while the $3.3 \mathrm{~V}$ battery was used to power the tag. A LM7805 regulator Integrated Circuit (IC) was used to convert the $9 \mathrm{~V}$ output from the battery into the $5 \mathrm{~V}$ needed by encoder and the transmitter. 


\begin{tabular}{|c|c|}
\hline \multicolumn{2}{|c|}{ Algorithm 1: Base station behaviour definition } \\
\hline Step 1 & Start \\
\hline Step 2 & Initialize $R F$ transmitter. \\
\hline Step 3 & Initialize HT12E encoder. \\
\hline Step 4 & $\begin{array}{l}\text { The RF transmitter generates analog signals } \\
\text { as output. }\end{array}$ \\
\hline Step 5 & $\begin{array}{l}\text { The generated wave occurs at the rate of } \\
1 \mathrm{Kbps}-10 \mathrm{Kbps} \text {. }\end{array}$ \\
\hline Step 6 & $\begin{array}{l}\text { The generated wave from the RF transmitter } \\
\text { is fed as input to the encoder. }\end{array}$ \\
\hline Step 7 & $\begin{array}{l}\text { The encoder starts a } 4 \text {-word transmission } \\
\text { cycle upon receiving transmission } \\
\text { enable signal. }\end{array}$ \\
\hline Step 8 & $\begin{array}{l}\text { The encoder repeats this output cycle as long } \\
\text { as transmission is enabled. }\end{array}$ \\
\hline Step 9 & $\begin{array}{l}\text { This loop continues for continuous data } \\
\text { transmission. }\end{array}$ \\
\hline Step 10 & End the operation. \\
\hline \multicolumn{2}{|r|}{ Algorithm 2: The tag subsystem definition } \\
\hline Step 1 & Start \\
\hline Step 2 & Initialize $R F$ receiver. \\
\hline Step 3 & Initialize HT12D decoder. \\
\hline Step 4 & $\begin{array}{l}\text { Oscillator is active when the decoder } \\
\text { receives data transmitted by the encoder. }\end{array}$ \\
\hline Step 5 & $\begin{array}{l}\text { Decoder starts decoding the received } \\
\text { address and data. }\end{array}$ \\
\hline Step 6 & $\begin{array}{l}\text { Decoder checks if the received address } \\
\text { matches three times continuously }\end{array}$ \\
\hline & $\begin{array}{l}\text { a) If no, the output pins are not } \\
\text { activated. }\end{array}$ \\
\hline & b) If yes, the output pins are activated. \\
\hline Step 7 & $\begin{array}{l}\text { The processed signal is then received by the } \\
R F \text { receiver. }\end{array}$ \\
\hline Step 8 & $\begin{array}{l}\text { The buzzer produces sound once the signal is } \\
\text { received by the RF receiver. }\end{array}$ \\
\hline Step 9 & $\begin{array}{l}\text { The data transmission is ended by the } R F \\
\text { transmitter. }\end{array}$ \\
\hline Step 10 & End the operation. \\
\hline
\end{tabular}

\subsubsection{Voltage regulator}

Voltage regulator was used to regulate power supply unit of the base station. It has the advantages of low cost and ease of use. It is a positive voltage regulator which produces a voltage that is positive relative to a common ground.

The whole process of implementation has been summarized and presented as Algorithm 1 and algorithm 2 in this paper. This is to facilitate proper understanding of the design process.

\subsection{Performance Evaluation}

The developed system was evaluated using detection-based metric. Detection based metric has been widely adopted in literature to obtain meaningful measures of the developed system's ability to perform object detection tasks. This metrics was also adopted in this work to evaluate the performance of the proposed ODS. This evaluation metric does not normally take into account the identities of objects over the lifespan of the test. When associating the lost object, six cases can occur which are zero-to-one, one-to-zero, oneto-one, many-to-one, one-to-many, and many-to-many associations. However, in this case, two cases can occur which are one-to-zero and one-to-one. One-to-zero case corresponds to no detection i.e. the lost item is not found while one-to-one corresponds to correct detection i.e. the lost item is found.

When the distance between the misplaced tagged items and the ODS base station was less or equal to 70 meters, there was not any case of one to zero, meaning one-to-one case was recorded to be $100 \%$. The case however exists after the distance between these two sub-systems goes beyond 70 meters. Also, it was observed that the strength of connection between the two sub-systems varies with the distance. Fig. 4 presents a description of the relationship between the distance in between the base station and the misplaced object. The curve shows that, the strength of the connection is inversely proportional to the distance between the two sub-systems.

\subsection{Comparison with existing systems}

The proposed ODS was compared to the existing systems available in market using the cost in market and the technology used. The result obtained on Table 1 shown that the proposed ODS system is the most cost effective and will even be far cheaper possibly within \$5-\$7 when produced in mass making it potentially affordable in market.

The proposed system has also considered the use of RF technology which makes it suitable to be used in most developing countries.

\section{CONCLUSION}

\subsection{Summary}

The system was designed, implemented and tested to ascertain the achievement of the set objectives. The system developed is capable of detecting lost objects and notify the user by triggering the buzzer in order to generate a sound which will be useful in tracing the lost item. The developed system is portable and can be used anywhere without any modification. Since the tag is not connected to the object, the tag can be removed and attach to another object if need demands.

\subsection{Future work}

This work has been able to use radio frequency to locate lost objects in homes. A lot of research still needed to be done on how to improve the system so as to obtain a more effective ODS. Future effort will include addition of some other functionalities such as ability to detect the proximity of the misplaced object from any mobile device before producing the system in mass. After this, efforts will be put in place to make the system available in market subject to availability of funding. Future works to improve the proposed ODS will therefore include:

a) The use of GSM module for more accurate reporting when the lost item is detected. This will allow users to receive perhaps on their mobile phones the location of the tagged object in a case where the sound is not enough to trace the lost item.

b) Use of a smaller battery for the tag in order to further reduce the size of the tag sub-system while making it more portable.

c) The tag sub-system should be designed in such a way that, it can be recharged without dismantling the system. 


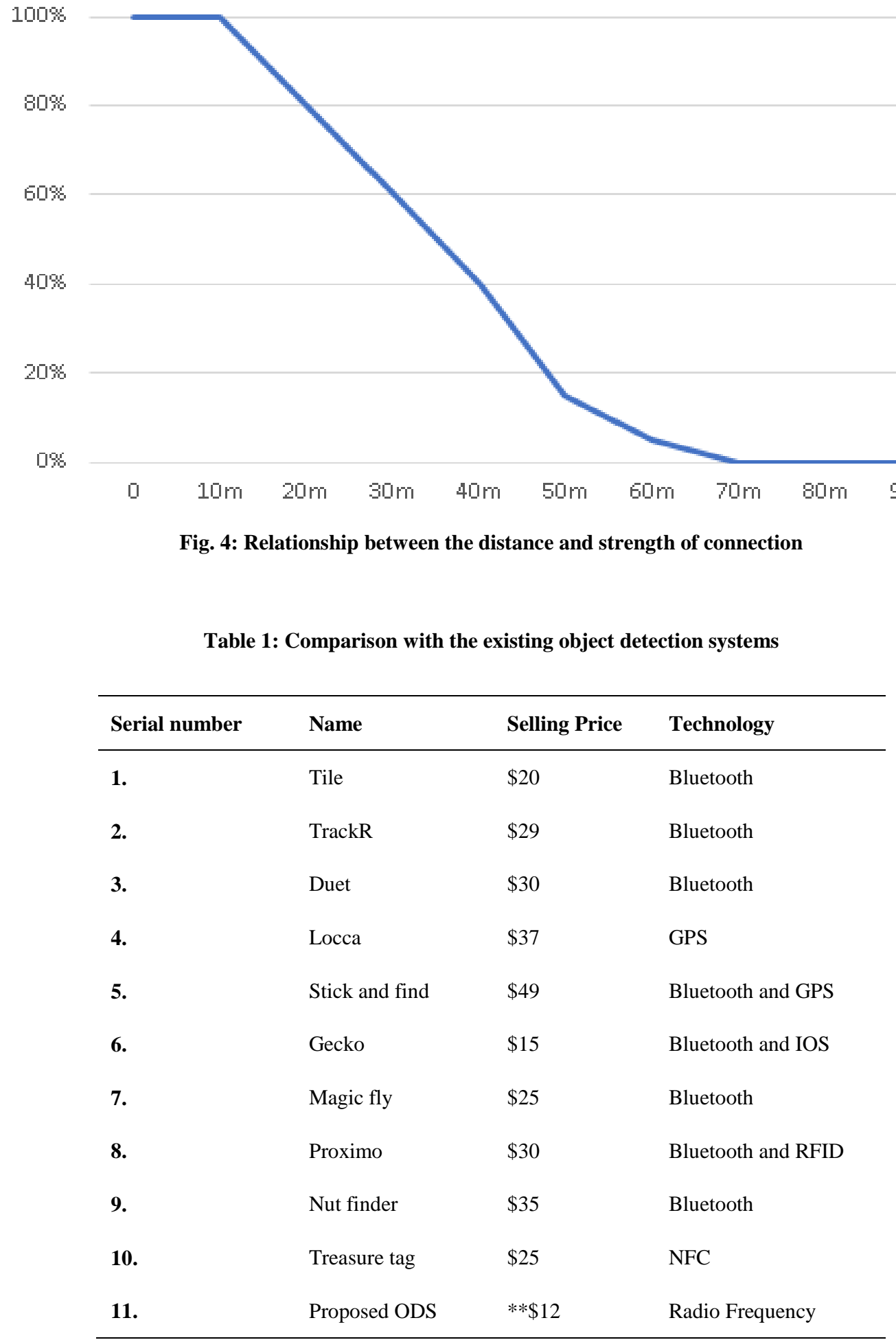

**Cost of implementing a single system NOT the selling price in market

\section{REFERENCES}

[1] Mowad, M. A. E. L., Fathy, A., and Hafez, A. (2014). Smart home automated control system using android application and microcontroller. International Journal of Scientific \& Engineering Research, 5(5), 935-939.

[2] George, A. (2010). U.S. Patent No. 7,844,368. Washington, DC: U.S. Patent and Trademark Office.
[3] Michael, G. G. (2004). U.S. Patent No. 6,802,471. Washington, DC: U.S. Patent and Trademark Office.

[4] Srinivasan, P., Shuvetha Antonia, U., and Anu Rekha, A. (2014). Locate Misplaced Objects.

[5] Nishigaki, M., and Saka, M. (2004). U.S. Patent No. 6,683,969. Washington, DC: US Patent and Trademark Office. 
[6] Frei, P., Mohler, E., Braun-Fahrländer, C., Fröhlich, J., Neubauer, G., and Röösli, M. (2012). Cohort study on the effects of everyday life radio frequency electromagnetic field exposure on non-specific symptoms and tinnitus. Environment international, 38(1), 29-36.

[7] Nakada, T., Kanai, H., and Kunifuji, S. (2005). A support system for finding lost objects using spotlight. In Proceedings of the 7th international conference on Human computer interaction with mobile devices \& services(pp. 321-322). ACM.

[8] Feldmann, S., Kyamakya, K., Zapater, A., and Lue, Z. (2014, June). An Indoor Bluetooth-Based Positioning System: Concept, Implementation and Experimental Evaluation. In International Conference on Wireless Networks (Vol. 272).
[9] Parkinson, W., and Runyon, J. (2007). U.S. Patent Application No. 11/111,790.

[10] Opoku, S. K. (2012). An indoor tracking system based on bluetooth technology. arXiv preprint arXiv:1209.3053.

[11] Sindhoori, S., and Kumar, N. S. Android based Secured Vehicle Key Finder System.

[12] Teller, E., King, M. T., Mannby, C. F., and Smith, M. J. (2014). U.S. Patent No. 8,810,392. Washington, DC: U.S. Patent and Trademark Office.

[13] Sarbani, D., Neha, K., Purushottam, S., Subhash, K., and Phiroz, S. (2016) "Object Detection and Tracking Robot using Android, Arduino and Open CV". International Journal of Computer Science and Mobile Computing, Vol.5 Issue.4, April- 2016. 